\title{
EFFECT OF KASNAZAN IMPOUNDMENT AND WELLWATER IN CHEMICAL PROPERTIES OF SOIL AND PLANT
}

Pakhshan Mustafa Maulood

Biology Dept., College of Sci., Salahaddin Univ.-Erbil, Iraq.

\begin{abstract}
Soil, plant, and water (Kasnazan impoundment and well water) samples were monthly collected from Kasnazan (10 Km north east of Erbil city) during August 2004 to February 2005. Chemical and statistical analysis indicated that the irrigation with well water resulted to decreases EC from $0.87 \mathrm{dS} . \mathrm{m}^{-1}$ to 0.49 $\mathrm{dS} . \mathrm{m}^{-1}$, because of leaching and dilution effects. Statistically there is a positive significant correlation $(\mathrm{P}<0.05)$ between $\mathrm{SAR}$ value of well water and irrigated soil with it, whereas, a negative correlation of $\mathrm{Mg}^{+2}$ concentrations observed between well water and soil irrigated with it. On the other hand, there was high content of $\mathrm{Mg}^{+2}$ and low content of $\mathrm{K}^{+1}$ for eucalyptus plant irrigated by well water in comparison to that irrigated with impoundment water.
\end{abstract}

\section{INTRODUCTION}

Water plays an important role in soil productivity and plant growth, and it is regarded as a limiting factor in plant growth. Much natural water contains impurities that make them directly harmful for plant. Plants vary in their tolerate to poor water qualities, so the soil regards as an important factor in limiting water suitability for irrigation (Taiz and Zeiger, 2006).

The quality of irrigation water is depended on salt content, the nature of salts present in solution and proportion of $\mathrm{Na}^{+}$to $\mathrm{Ca}^{+2}, \mathrm{Mg}^{+2}$ and other cations (Shirokova et al., 2000).

Iraqi Kurdistan Region is rich in water resources like rivers, streams, spring, lake, and impoundment water. Many limnological and phycological studies were conducted in Kasnazan impoundment water a large lentic system within Erbil province (Rashhed, 1994; Al-Barazingy, 1995; Toma, 2000; Bapeer, 2004 and Goran, 2006), whereas, there is a shortage information about water quality for irrigation purposes. This study is the first attempt to reduce existing gap about Kasnazan water quality and its effect on each of soil's ionic component and their effect on Eucalyptus camaldulensis (Dehn), in addition to comparing it with the result of ground water of Kasnazan location.

According to United State Salinity laboratory Staff (1954) Classified irrigation water to sixteen classes depending on $\mathrm{dS} . \mathrm{m}^{-1}$ at $25^{\circ} \mathrm{C}$ and SAR as follows:

\begin{tabular}{|c|c|}
\hline Water Classes & Electrical Conductivity dS.m ${ }^{-1}$ at $25{ }^{\circ} \mathrm{C}$ \\
\hline C1 Low- salinity & $0<\mathrm{EC}<0.25$ \\
\hline C2 Medium- salinity & $0.25<\mathrm{EC}<0.75$ \\
\hline C3 High - salinity & $0.75<\mathrm{EC}<2.25$ \\
\hline C4 Very high -salinity & $2.25<\mathrm{EC}<5$ \\
\hline \multicolumn{2}{|c|}{ Continued } \\
\hline Water Classes & SAR \\
\hline S1= Excellent & $<10$ \\
\hline S2= Good & $10-18$ \\
\hline S3 = Fair & $18-26$ \\
\hline
\end{tabular}



an altitude of $646 \mathrm{~m}$, latitude $38{ }^{\circ} \mathrm{S} 0423768$, and longitude $40^{\circ} 07776$ UTM(Goran, 2006). Its artificial water basin instructed to collect water from the perennial Kahreez. The water is used for various purposes: drinking, domestic uses, irrigation, and swimming, whereas, orchard field is located at eastern side of Kasnazan village about $2 \mathrm{Km}$ far from water impoundment, in which irrigated by well water.

Water, surface soil $(0-30 \mathrm{~cm})$ and plant samples (Eucalyptus sp.) were collected during August 2004 to February 2005 from Kasnazan village (which irrigated by impoundment water), as well as, from orchard field. $\mathrm{EC}, \mathrm{pH}$ and TDS were measured by using (pH-EC-TDS meter, HI 9812, Hanna Instrument). Phosphorus and total nitrogen were estimated by (Rayan et al., 2001). Nitrate was measured by using (Nitrachech 404, Q40 Med. Ltd Instument). Calcium, magnesium, Sodium, potassium, bicarbonate, carbonate, and chloride were estimated according to Page et al. (1982). Data were statistically analysis using SPSS program.

\section{RESULTS AND DISCUSSION}

Table(1) shows some chemical properties of Kasnazan impoundment water and well water, the $\mathrm{pH}$ values were range from 7.30 to 8.20 and 6.70 to 8.20 respectively, while the SAR values for Kasnazan impoundment water were range from 0.15 to 1.53 in comparing with 0.07 to 0.11 for well water, this may be attributed to high $\mathrm{Na}^{+}$concentration and low $\mathrm{Ca}^{+2}$ and $\mathrm{Mg}^{+2}$ concentrations in Kasnazan impoundment water in comparing with low $\mathrm{Na}^{+1}$ concentration and high $\mathrm{Ca}^{+2}$ and $\mathrm{Mg}^{+2}$ concentrations in the well water (Shirokova et al., 2000). Depending on US Salinity Laboratory (1954) Kasnazan water impoundment and well water can be classified as C1S1 class according to values of SAR and EC. On the other hand, significant differences $(\mathrm{P}<0.05)$ were observed depending $\mathrm{SAR}$ value in soil irrigated by well water. The present results were relatively similar to that of Esmael et al. (2007) in Kasnazan water impoundment, with maximum $\mathrm{Na}^{+1}$ concentration 0.356 Meq. $\mathrm{l}^{-}$ ${ }^{1}$, they reported that the dominant cation was $\mathrm{Na}^{+1}$ while the dominant anion was $\mathrm{Cl}^{-1}$, this was contrast to other studies conducted in the area with references to the dominance of $\mathrm{Ca}^{+2}$ and $\mathrm{HCO}^{-}$in water (Esmael, 1986; Dohuki, 1997 and Goran, 2006). Although, the maximum concentrations of $\mathrm{Ca}^{+2}$ and $\mathrm{Mg}^{+2}$ for Kasnazan impoundment and well water were 0.14 and 6.40 Meq. $\mathrm{l}^{-1}$ and 0.25 and 10.2 Meq. $\mathrm{l}^{-1}$ respectively. Furthermore, the maximum concentrations of $\mathrm{Cl}^{-}$were 0.53 and 0.52 Meq. $1^{-1}$ for Kasnazan water impoundment and well water respectively. According to Van Hoorn (1970) both analyzed water were suitable for irrigation of all types of plants. Meanwhile, the maximum SSPP value for Kasnazan water impoundment was 0.75, which was lower than that obtain by Esmael et al.(2007) in the same water impoundment. 
Table (2) indicates the effect of irrigation with well water on some chemical properties of the soil comparison to non irrigated soil, it showed that the irrigation causes a decrease in EC value; this may be due to leaching and dilution effect (Page et al., 1982). Statistically there was a positive significant correlation between SAR value of well water and irrigated soil $(\mathrm{P}<0.05)$, this may be attributed to increasing in $\mathrm{Na}^{+1}$ concentration of soil after irrigation (Guo L, 2003). Whereas, a negative correlation of $\mathrm{Mg}^{+2}$ concentrations ( $\mathrm{P}<$ 0.05) was observed between well water and irrigated soil.

Irrigation with impoundment water caused a reduction in EC from 1.27 to $0.80 \mathrm{dS} . \mathrm{m}^{-1}$ (as mean value); this may be due to leaching effect (table 3), (Page et al., 1982). The comparison test (paired t-test) between soil irrigated with impoundment water and well water showed a significant correlation between concentration of $\mathrm{Mg}^{+2}$ ion in both cases that may be due to water family which belong to (Mg-Cl) family.(Esmael, 1986).

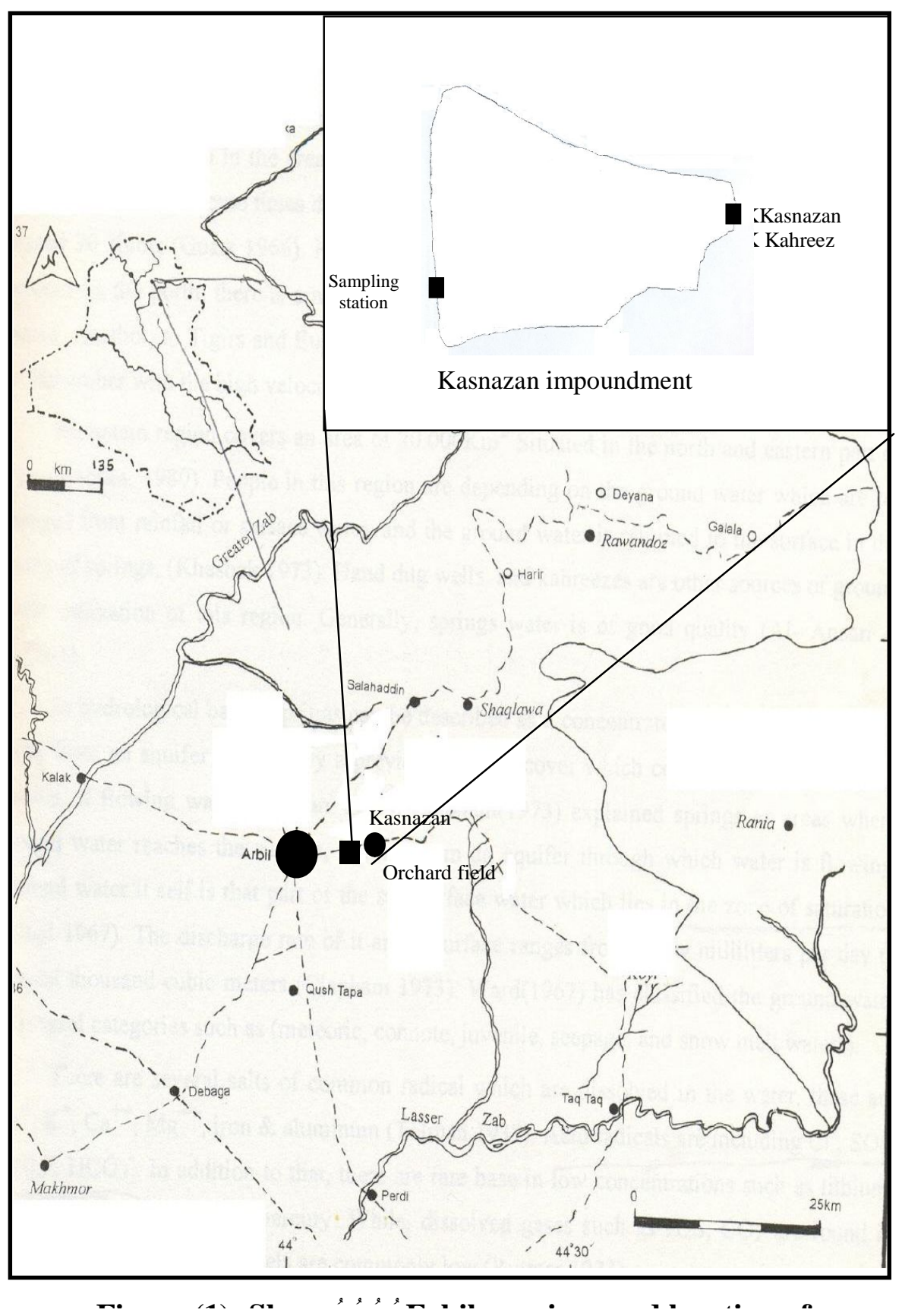


Table (1):Some chemical properties of Kasnazan water impoundment (upper numbers) and well water(between brackets) used for irrigation.

\begin{tabular}{|c|c|c|c|c|c|c|c|c|c|c|c|c|c|}
\hline Date & $\mathrm{pH}$ & $\mathrm{EC} \mathrm{dS.m^{-1 }}$ & $\begin{array}{c}\mathrm{PO}_{4} \\
\mathrm{mg} .^{-1}\end{array}$ & $\begin{array}{c}\mathrm{NO}_{3} \\
{\mathrm{mg} .1^{-1}}^{-1}\end{array}$ & $\begin{array}{c}\mathrm{Na}^{+} \\
\text {Meq. } .^{-1}\end{array}$ & $\begin{array}{c}\mathrm{K}^{+} \\
\text {Meq. } .^{-1}\end{array}$ & $\begin{array}{c}\mathrm{Ca}^{+2} \\
\text { Meq. } .^{-1}\end{array}$ & $\begin{array}{c}\mathrm{Mg}^{+2} \\
\text { Meq.1 }\end{array}$ & $\begin{array}{l}\mathrm{HCO}_{3}{ }^{-} \\
\mathrm{Meq}^{-1} \mathrm{l}^{-1}\end{array}$ & $\begin{array}{c}\mathrm{CO}_{3}{ }^{-2} \\
\text { Meq. } .^{-1}\end{array}$ & $\mathrm{Cl}^{-}$Meq..$^{-1}$ & SAR & SSPP \\
\hline $8-2004$ & $\begin{array}{c}7.3 \\
(6.8)\end{array}$ & $\begin{array}{c}0.36 \\
(0.45)\end{array}$ & $\begin{array}{l}0.17 \\
(\mathrm{ND})\end{array}$ & $\begin{array}{c}11 \\
(12)\end{array}$ & $\begin{array}{c}0.43 \\
(0.25)\end{array}$ & $\begin{array}{c}0.06 \\
(0.02)\end{array}$ & $\begin{array}{c}0.12 \\
(5.55)\end{array}$ & $\begin{array}{c}0.12 \\
(5.92)\end{array}$ & $\begin{array}{c}0.04 \\
(0.04)\end{array}$ & $\begin{array}{c}0 \\
(0)\end{array}$ & $\begin{array}{c}0.53 \\
(0.41)\end{array}$ & $\begin{array}{c}1.23 \\
(0.11)\end{array}$ & $\begin{array}{c}0.67 \\
(0.02)\end{array}$ \\
\hline $9-2004$ & $\begin{array}{c}8.0 \\
(8.2)\end{array}$ & $\begin{array}{c}0.33 \\
(0.32)\end{array}$ & $\begin{array}{l}0.36 \\
\text { (ND) }\end{array}$ & $\begin{array}{l}9.0 \\
(11)\end{array}$ & $\begin{array}{c}0.45 \\
(0.26)\end{array}$ & $\begin{array}{c}0.29 \\
(0.04)\end{array}$ & $\begin{array}{c}0.08 \\
(5.05)\end{array}$ & $\begin{array}{c}0.13 \\
(5.42)\end{array}$ & $\begin{array}{c}0.03 \\
(0.03)\end{array}$ & $\begin{array}{c}0 \\
(0)\end{array}$ & $\begin{array}{c}0.31 \\
(0.34)\end{array}$ & $\begin{array}{c}1.41 \\
(0.11)\end{array}$ & $\begin{array}{c}0.72 \\
(0.02)\end{array}$ \\
\hline $10-2004$ & $\begin{array}{c}8.0 \\
(7.1)\end{array}$ & $\begin{array}{c}0.25 \\
(0.41)\end{array}$ & $\begin{array}{c}0.34 \\
(\mathrm{ND})\end{array}$ & $\begin{array}{c}22 \\
(13)\end{array}$ & $\begin{array}{c}0.44 \\
(0.27)\end{array}$ & $\begin{array}{c}0.06 \\
(0.02)\end{array}$ & $\begin{array}{c}0.04 \\
(5.30)\end{array}$ & $\begin{array}{c}0.13 \\
(6.90)\end{array}$ & $\begin{array}{c}0.02 \\
(0.04)\end{array}$ & $\begin{array}{c}0 \\
(0)\end{array}$ & $\begin{array}{c}0.41 \\
(0.41)\end{array}$ & $\begin{array}{c}1.53 \\
(0.11)\end{array}$ & $\begin{array}{c}0.75 \\
(0.02)\end{array}$ \\
\hline $11-2004$ & $\begin{array}{c}8.2 \\
(7.1)\end{array}$ & $\begin{array}{c}0.36 \\
(0.45)\end{array}$ & $\begin{array}{l}0.00 \\
(\mathrm{ND})\end{array}$ & $\begin{array}{c}13 \\
(9.0)\end{array}$ & $\begin{array}{c}0.190 \\
(0.15)\end{array}$ & $\begin{array}{c}0.06 \\
(0.04)\end{array}$ & $\begin{array}{c}0.06 \\
(2.34)\end{array}$ & $\begin{array}{c}0.13 \\
(8.05)\end{array}$ & $\begin{array}{c}0.02 \\
(0.05)\end{array}$ & $\begin{array}{c}0 \\
(0)\end{array}$ & $\begin{array}{c}0.33 \\
(0.52)\end{array}$ & $\begin{array}{c}0.64 \\
(0.07)\end{array}$ & $\begin{array}{c}0.54 \\
(0.01)\end{array}$ \\
\hline $12-2004$ & $\begin{array}{c}7.6 \\
(6.7)\end{array}$ & $\begin{array}{c}0.56 \\
(0.44)\end{array}$ & $\begin{array}{l}0.00 \\
(\mathrm{ND})\end{array}$ & $\begin{array}{c}11 \\
(18)\end{array}$ & $\begin{array}{c}0.31 \\
(0.20)\end{array}$ & $\begin{array}{c}0.06 \\
(0.05)\end{array}$ & $\begin{array}{c}0.06 \\
(4.85)\end{array}$ & $\begin{array}{c}0.25 \\
(10.1)\end{array}$ & $\begin{array}{c}0.04 \\
(0.04)\end{array}$ & $\begin{array}{c}0 \\
(0)\end{array}$ & $\begin{array}{c}0.36 \\
(0.41)\end{array}$ & $\begin{array}{c}0.78 \\
(0.07) \\
\end{array}$ & $\begin{array}{r}0.53 \\
(0.01) \\
\end{array}$ \\
\hline $1-2005$ & $\begin{array}{c}8.1 \\
(7.5)\end{array}$ & $\begin{array}{c}0.64 \\
(0.45) \\
\end{array}$ & $\begin{array}{l}0.02 \\
(\mathrm{ND})\end{array}$ & $\begin{array}{c}8.0 \\
(7.0)\end{array}$ & $\begin{array}{c}0.09 \\
(0.20) \\
\end{array}$ & $\begin{array}{c}0.02 \\
(0.05)\end{array}$ & $\begin{array}{c}0.12 \\
(6.40) \\
\end{array}$ & $\begin{array}{c}0.18 \\
(8.14) \\
\end{array}$ & $\begin{array}{c}0.04 \\
(0.04) \\
\end{array}$ & $\begin{array}{c}0 \\
(0)\end{array}$ & $\begin{array}{c}0.30 \\
(0.25) \\
\end{array}$ & $\begin{array}{c}0.24 \\
(0.08) \\
\end{array}$ & $\begin{array}{c}0.26 \\
(0.01) \\
\end{array}$ \\
\hline $2-2005$ & $\begin{array}{c}7.9 \\
(7.5)\end{array}$ & $\begin{array}{c}0.66 \\
(0.43)\end{array}$ & $\begin{array}{l}0.02 \\
\text { (ND) }\end{array}$ & $\begin{array}{c}17 \\
(25)\end{array}$ & $\begin{array}{c}0.28 \\
(0.21)\end{array}$ & $\begin{array}{c}0.06 \\
(0.04)\end{array}$ & $\begin{array}{c}0.14 \\
(6.15)\end{array}$ & $\begin{array}{c}0.16 \\
(10.2)\end{array}$ & $\begin{array}{c}0.04 \\
(0.04)\end{array}$ & $\begin{array}{c}0 \\
(0)\end{array}$ & $\begin{array}{c}0.33 \\
(0.36)\end{array}$ & $\begin{array}{c}0.15 \\
(0.07)\end{array}$ & $\begin{array}{c}0.51 \\
(0.01)\end{array}$ \\
\hline
\end{tabular}

$\mathrm{ND}=$ non detected 
Table (2):-Some chemical properties of non irrigated field soil (upper numbers) and irrigated soil (between brackets) by well water.

\begin{tabular}{|c|c|c|c|c|c|c|c|c|c|c|c|c|}
\hline Date & $\mathrm{pH}$ & $\mathrm{EC} \mathrm{dS} . \mathrm{m}^{-1}$ & $\begin{array}{c}\text { Total } \\
\text { phosphorus } \\
\%\end{array}$ & $\begin{array}{c}\text { Total } \\
\text { nitrogen } \\
\%\end{array}$ & $\mathrm{Na}^{+}$Meq..$^{-1}$ & $\begin{array}{c}\mathrm{K}^{+} \\
\text {Meq. } 1^{-1}\end{array}$ & $\begin{array}{c}\mathrm{Ca}^{+2} \\
\text { Meq. } .^{-1}\end{array}$ & $\begin{array}{c}\mathrm{Mg}^{+2} \\
\text { Meq. } .^{-1}\end{array}$ & $\begin{array}{l}\mathrm{HCO}_{3}{ }^{-} \\
\text {Meq. }^{-1}\end{array}$ & $\begin{array}{l}\mathrm{CO}_{3}{ }^{-2} \\
\text { Meq. } .^{-1}\end{array}$ & $\begin{array}{c}\mathrm{Cl}^{-} \\
\text {Meq. } \mathrm{l}^{-1}\end{array}$ & SAR \\
\hline $8-2004$ & $\begin{array}{c}7.0 \\
(7.4)\end{array}$ & $\begin{array}{c}0.93 \\
(0.40)\end{array}$ & $\begin{array}{c}0.032 \\
(0.022)\end{array}$ & $\begin{array}{c}0.35 \\
(0.28)\end{array}$ & $\begin{array}{c}10.3 \\
(8.45)\end{array}$ & $\begin{array}{c}17.4 \\
(17.3)\end{array}$ & $\begin{array}{c}3.60 \\
(2.80)\end{array}$ & $\begin{array}{c}28.0 \\
(27.4)\end{array}$ & $\begin{array}{c}3.20 \\
(4.60)\end{array}$ & $\begin{array}{c}0 \\
(0)\end{array}$ & $\begin{array}{c}2.20 \\
(2.40)\end{array}$ & $\begin{array}{c}2.60 \\
(2.17)\end{array}$ \\
\hline $9-2004$ & $\begin{array}{c}7.1 \\
(7.3)\end{array}$ & $\begin{array}{c}0.58 \\
(0.28)\end{array}$ & $\begin{array}{c}0.029 \\
(0.017)\end{array}$ & $\begin{array}{c}0.27 \\
(0.32)\end{array}$ & $\begin{array}{c}8.45 \\
(21.1)\end{array}$ & $\begin{array}{c}18.3 \\
(19.7)\end{array}$ & $\begin{array}{c}2.40 \\
(4.00)\end{array}$ & $\begin{array}{c}25.2 \\
(24.6)\end{array}$ & $\begin{array}{c}3.20 \\
(4.80)\end{array}$ & $\begin{array}{c}0 \\
(0)\end{array}$ & $\begin{array}{c}2.20 \\
(2.60)\end{array}$ & $\begin{array}{c}2.27 \\
(5.58)\end{array}$ \\
\hline $10-2004$ & $\begin{array}{c}7.7 \\
(7.5)\end{array}$ & $\begin{array}{c}0.77 \\
(0.40)\end{array}$ & $\begin{array}{c}0.025 \\
(0.017)\end{array}$ & $\begin{array}{c}0.16 \\
(0.20)\end{array}$ & $\begin{array}{c}7.04 \\
(7.51)\end{array}$ & $\begin{array}{c}16.0 \\
(19.3)\end{array}$ & $\begin{array}{c}2.60 \\
(3.60)\end{array}$ & $\begin{array}{c}22.0 \\
(21.2)\end{array}$ & $\begin{array}{c}4.40 \\
(4.00)\end{array}$ & $\begin{array}{c}0 \\
(0)\end{array}$ & $\begin{array}{c}2.20 \\
(2.60)\end{array}$ & $\begin{array}{c}2.01 \\
(2.13)\end{array}$ \\
\hline $11-2004$ & $\begin{array}{c}7.4 \\
(7.2)\end{array}$ & $\begin{array}{c}0.50 \\
(0.68)\end{array}$ & $\begin{array}{c}0.026 \\
(0.019)\end{array}$ & $\begin{array}{c}0.25 \\
(0.34)\end{array}$ & $\begin{array}{c}9.86 \\
(9.39)\end{array}$ & $\begin{array}{c}18.3 \\
(11.7)\end{array}$ & $\begin{array}{c}2.60 \\
(3.00)\end{array}$ & $\begin{array}{c}18.0 \\
(21.6)\end{array}$ & $\begin{array}{c}4.60 \\
(5.60)\end{array}$ & $\begin{array}{c}0 \\
(0)\end{array}$ & $\begin{array}{c}1.40 \\
(2.60)\end{array}$ & $\begin{array}{c}3.07 \\
(2.68)\end{array}$ \\
\hline $12-2004$ & $\begin{array}{c}7.6 \\
(7.4)\end{array}$ & $\begin{array}{c}1.41 \\
(0.45)\end{array}$ & $\begin{array}{c}0.035 \\
(0.025)\end{array}$ & $\begin{array}{c}0.25 \\
(0.36)\end{array}$ & $\begin{array}{c}12.2 \\
(15.0)\end{array}$ & $\begin{array}{c}17.9 \\
(18.8)\end{array}$ & $\begin{array}{c}3.60 \\
(2.60)\end{array}$ & $\begin{array}{c}19.2 \\
(21.6)\end{array}$ & $\begin{array}{c}4.40 \\
(4.58)\end{array}$ & $\begin{array}{c}0 \\
(0)\end{array}$ & $\begin{array}{c}2.00 \\
(1.40)\end{array}$ & $\begin{array}{c}3.62 \\
(4.32)\end{array}$ \\
\hline $1-2005$ & $\begin{array}{c}7.2 \\
(7.1)\end{array}$ & $\begin{array}{c}0.82 \\
(0.66)\end{array}$ & $\begin{array}{c}0.034 \\
(0.026)\end{array}$ & $\begin{array}{c}0.16 \\
(0.24)\end{array}$ & $\begin{array}{c}9.39 \\
(17.8)\end{array}$ & $\begin{array}{c}16.0 \\
(18.8)\end{array}$ & $\begin{array}{c}3.60 \\
(1.60)\end{array}$ & $\begin{array}{c}21.8 \\
(22.8)\end{array}$ & $\begin{array}{c}4.80 \\
(4.80)\end{array}$ & $\begin{array}{c}0 \\
(0)\end{array}$ & $\begin{array}{c}1.20 \\
(1.00)\end{array}$ & $\begin{array}{c}2.63 \\
(5.11)\end{array}$ \\
\hline $2-2005$ & $\begin{array}{c}7.4 \\
(6.6)\end{array}$ & $\begin{array}{c}1.09 \\
(0.55)\end{array}$ & $\begin{array}{c}0.027 \\
(0.028)\end{array}$ & $\begin{array}{c}0.17 \\
(0.30)\end{array}$ & $\begin{array}{c}11.7 \\
(19.3)\end{array}$ & $\begin{array}{c}15.5 \\
(17.4)\end{array}$ & $\begin{array}{c}2.60 \\
(1.60)\end{array}$ & $\begin{array}{c}17.8 \\
(18.6)\end{array}$ & $\begin{array}{c}4.80 \\
(5.00)\end{array}$ & $\begin{array}{c}0 \\
0 \\
(0)\end{array}$ & $\begin{array}{c}2.20 \\
(1.40)\end{array}$ & $\begin{array}{c}3.68 \\
(6.07)\end{array}$ \\
\hline
\end{tabular}


Table (3):-Some chemical properties of non irrigated Kasnazan soil (upper numbers) and irrigated soil (between brackets) by Kasnazan water impoundment.

\begin{tabular}{|c|c|c|c|c|c|c|c|c|c|c|c|c|}
\hline Date & $\mathrm{pH}$ & $\mathrm{EC} \mathrm{dS} \cdot \mathrm{m}^{-1}$ & $\begin{array}{c}\text { Total } \\
\text { phosphorus } \\
\%\end{array}$ & $\begin{array}{c}\text { Total } \\
\text { nitrogen } \\
\%\end{array}$ & $\mathrm{Na}^{+}$Meq..$^{-1}$ & $\begin{array}{c}\mathrm{K}^{+} \\
\text {Meq. } .^{-1}\end{array}$ & $\begin{array}{c}\mathrm{Ca}^{+2} \\
\text { Meq. } .^{-1}\end{array}$ & $\begin{array}{c}\mathrm{Mg}^{+2} \\
\text { Meq. }^{-1}\end{array}$ & $\begin{array}{l}\mathrm{HCO}_{3}{ }^{-} \\
\text {Meq. } .^{-1}\end{array}$ & $\begin{array}{c}\mathrm{CO}_{3}{ }^{-2} \\
\text { Meq. } .^{-1}\end{array}$ & $\begin{array}{c}\mathrm{Cl}^{-} \\
\text {Meq. } .^{-1}\end{array}$ & SAR \\
\hline $8-2004$ & $\begin{array}{c}7.3 \\
(7.8)\end{array}$ & $\begin{array}{c}1.58 \\
(1.27)\end{array}$ & $\begin{array}{c}0.028 \\
(0.026)\end{array}$ & $\begin{array}{c}0.21 \\
(0.23)\end{array}$ & $\begin{array}{c}8.92 \\
(18.3)\end{array}$ & $\begin{array}{c}9.89 \\
(11.7)\end{array}$ & $\begin{array}{c}2.60 \\
(1.60)\end{array}$ & $\begin{array}{c}19.2 \\
(20.8)\end{array}$ & $\begin{array}{c}6.00 \\
(6.00)\end{array}$ & $\begin{array}{c}0 \\
(0)\end{array}$ & $\begin{array}{c}1.80 \\
(1.60)\end{array}$ & $\begin{array}{c}2.70 \\
(5.48)\end{array}$ \\
\hline $9-2004$ & $\begin{array}{c}7.2 \\
(7.3)\end{array}$ & $\begin{array}{c}3.09 \\
(0.66)\end{array}$ & $\begin{array}{c}0.027 \\
(0.025)\end{array}$ & $\begin{array}{c}0.24 \\
(0.33)\end{array}$ & $\begin{array}{c}11.3 \\
(17.3)\end{array}$ & $\begin{array}{c}20.3 \\
(10.8)\end{array}$ & $\begin{array}{c}3.60 \\
(1.00)\end{array}$ & $\begin{array}{c}19.0 \\
(19.2)\end{array}$ & $\begin{array}{c}8.40 \\
(5.20)\end{array}$ & $\begin{array}{c}0 \\
(0)\end{array}$ & $\begin{array}{c}2.00 \\
(1.40)\end{array}$ & $\begin{array}{c}2.36 \\
(5.47)\end{array}$ \\
\hline $10-2004$ & $\begin{array}{c}7.6 \\
(7.2)\end{array}$ & $\begin{array}{c}2.36 \\
(1.05)\end{array}$ & $\begin{array}{c}0.028 \\
(0.022)\end{array}$ & $\begin{array}{c}0.27 \\
(0.35)\end{array}$ & $\begin{array}{c}12.2 \\
(20.1)\end{array}$ & $\begin{array}{c}17.0 \\
(11.7)\end{array}$ & $\begin{array}{c}4.20 \\
(3.00)\end{array}$ & $\begin{array}{c}18.4 \\
(13.2)\end{array}$ & $\begin{array}{c}8.80 \\
(5.20)\end{array}$ & $\begin{array}{c}0 \\
(0)\end{array}$ & $\begin{array}{c}2.00 \\
(1.40)\end{array}$ & $\begin{array}{c}3.63 \\
(7.10)\end{array}$ \\
\hline $11-2004$ & $\begin{array}{c}7.4 \\
(7.3)\end{array}$ & $\begin{array}{c}0.47 \\
(0.39)\end{array}$ & $\begin{array}{c}0.031 \\
(0.017)\end{array}$ & $\begin{array}{c}0.25 \\
(0.24)\end{array}$ & $\begin{array}{c}10.3 \\
(17.8)\end{array}$ & $\begin{array}{c}11.8 \\
(13.6)\end{array}$ & $\begin{array}{c}4.00 \\
(3.60)\end{array}$ & $\begin{array}{c}25.6 \\
(11.4)\end{array}$ & $\begin{array}{c}7.60 \\
(5.20)\end{array}$ & $\begin{array}{c}0 \\
(0)\end{array}$ & $\begin{array}{c}2.60 \\
(1.00)\end{array}$ & $\begin{array}{c}2.69 \\
(6.53)\end{array}$ \\
\hline $12-2004$ & $\begin{array}{c}7.2 \\
(7.0)\end{array}$ & $\begin{array}{c}0.57 \\
(1.26)\end{array}$ & $\begin{array}{c}0.029 \\
(0.020)\end{array}$ & $\begin{array}{c}0.27 \\
(0.45)\end{array}$ & $\begin{array}{c}9.86 \\
(19.7)\end{array}$ & $\begin{array}{c}13.7 \\
(8.48)\end{array}$ & $\begin{array}{c}3.60 \\
(3.60)\end{array}$ & $\begin{array}{c}26.0 \\
(12.6)\end{array}$ & $\begin{array}{c}6.00 \\
(4.80)\end{array}$ & $\begin{array}{c}0 \\
(0)\end{array}$ & $\begin{array}{c}1.80 \\
(1.60)\end{array}$ & $\begin{array}{c}2.56 \\
(6.94) \\
\end{array}$ \\
\hline $1-2005$ & $\begin{array}{c}7.3 \\
(7.4)\end{array}$ & $\begin{array}{c}0.45 \\
(0.35)\end{array}$ & $\begin{array}{c}0.020 \\
(0.026)\end{array}$ & $\begin{array}{c}0.25 \\
(0.28)\end{array}$ & $\begin{array}{c}7.51 \\
(17.8)\end{array}$ & $\begin{array}{c}8.48 \\
(14.6)\end{array}$ & $\begin{array}{c}3.60 \\
(3.60)\end{array}$ & $\begin{array}{c}19.0 \\
(12.6)\end{array}$ & $\begin{array}{c}4.80 \\
(4.40)\end{array}$ & $\begin{array}{c}0 \\
(0)\end{array}$ & $\begin{array}{c}2.60 \\
(1.80)\end{array}$ & $\begin{array}{c}2.23 \\
(6.28)\end{array}$ \\
\hline $2-2005$ & $\begin{array}{c}7.1 \\
(7.4)\end{array}$ & $\begin{array}{c}0.37 \\
(0.63)\end{array}$ & $\begin{array}{c}0.021 \\
90.028)\end{array}$ & $\begin{array}{c}0.31 \\
(0.30)\end{array}$ & $\begin{array}{c}8.45 \\
(19.2)\end{array}$ & $\begin{array}{c}16.5 \\
(10.3)\end{array}$ & $\begin{array}{c}3.60 \\
(3.20)\end{array}$ & $\begin{array}{c}23.2 \\
(13.8)\end{array}$ & $\begin{array}{c}4.40 \\
(7.60)\end{array}$ & $\begin{array}{c}0 \\
(0)\end{array}$ & $\begin{array}{c}2.20 \\
(1.60)\end{array}$ & $\begin{array}{c}2.30 \\
(6.61)\end{array}$ \\
\hline
\end{tabular}


As shown in table(4), $\mathrm{Mg}^{+2}$ content of Eucalyptus plant irrigated with well water was higher than that irrigated with impoundment water, this may be due to high concentration of $\mathrm{Mg}^{+2}$ in well water in comparing with its concentration in impoundment water (table 1). But in the case of $\mathrm{K}^{+}$content of plant the opposite result was recorded which may be due to the high $\mathrm{K}^{+}$ concentration in impoundment water comparing with well water (Abu-Thahe, 1989).

Although, negative correlation of phosphorus concentration were observed between non-irrigated soil by impoundment water and than that irrigated by impoundment water, which may resulted in increasing of phosphorus content of Eucalyptos plant irrigated by impoundment water. Esmael (1986) Stated that increasing of $\mathrm{Mg}^{+2}$ concentrations in soil solution exceeded phosphorus absorption by plant. On the other hand, $\mathrm{Na}^{+}$concentration followed the same pattern of phosphorus, in which significant differences $(\mathrm{P}<0.05)$ were observed between plant irrigated either by water impoundment than well water, as well as, for $\mathrm{K}^{+}$plant content. Dohuki (1997) noticed that increase in $\mathrm{Na}^{+}$concentration causes increase in phosphorus availability leading to increase in phosphorus content of plant.

Finally, additional researches using different plants for longer periods are needed to obtain more results and information.

Table (4):Ionic and nutrient contents of Eucalyptus tree irrigated by Kasnazan Impoundment water (upper numbers) and well water(between brackets).

\begin{tabular}{|c|c|c|c|c|c|c|c|}
\hline Date & $\begin{array}{c}\text { Total } \\
\text { phosphorus } \\
\text { mg.g }\end{array}$ & $\begin{array}{c}\text { Total } \\
\text { nitrogen } \\
\%\end{array}$ & $\begin{array}{c}\mathrm{Na}^{+} \\
\mathrm{mg} \cdot \mathrm{g}^{-1}\end{array}$ & $\begin{array}{c}\mathrm{K}^{+} \\
\mathrm{mg} \cdot \mathrm{g}^{-1}\end{array}$ & $\begin{array}{c}\mathrm{Ca}^{+2} \\
\mathrm{mg}^{-1}\end{array}$ & $\begin{array}{c}\mathrm{Mg}^{+2} \\
\mathrm{mg}^{-1}\end{array}$ & $\begin{array}{c}\mathrm{Cl}^{-} \\
\mathrm{mg} \cdot \mathrm{g}^{-1}\end{array}$ \\
\hline $8-2004$ & $\begin{array}{c}0.22 \\
(0.29)\end{array}$ & $\begin{array}{c}1.06 \\
(0.73)\end{array}$ & $\begin{array}{c}0.54 \\
(0.60)\end{array}$ & $\begin{array}{c}1.34 \\
(0.31)\end{array}$ & $\begin{array}{c}0.07 \\
(0.06)\end{array}$ & $\begin{array}{c}0.14 \\
(0.07)\end{array}$ & $\begin{array}{c}0.07 \\
(0.06)\end{array}$ \\
\hline $9-2004$ & $\begin{array}{c}0.28 \\
(0.30)\end{array}$ & $\begin{array}{c}1.23 \\
(1.15)\end{array}$ & $\begin{array}{c}0.44 \\
(0.84)\end{array}$ & $\begin{array}{c}1.51 \\
(0.83)\end{array}$ & $\begin{array}{c}0.05 \\
(0.06)\end{array}$ & $\begin{array}{c}0.18 \\
(0.16)\end{array}$ & $\begin{array}{c}0.06 \\
(0.07)\end{array}$ \\
\hline $10-2004$ & 0.28 & 1.27 & 0.47 & 1.29 & 0.06 & 0.17 & 0.05 \\
& $(0.30)$ & $(1.24)$ & $(0.84)$ & $(0.98)$ & $(0.08)$ & $(0.17)$ & $(0.04)$ \\
\hline $11-2004$ & 0.26 & 1.07 & 0.43 & 1.47 & 0.08 & 0.03 & 0.04 \\
& $(0.25)$ & $(1.05)$ & $(0.79)$ & $(0.81)$ & $(0.08)$ & $(0.15)$ & $(0.04)$ \\
\hline $12-2004$ & 0.19 & 0.82 & 0.43 & 1.16 & 0.06 & 0.04 & 0.06 \\
& $(0.25)$ & $(1.01)$ & $(0.79)$ & $(0.72)$ & $(0.06)$ & $(0.20)$ & $(0.06)$ \\
\hline $1-2005$ & 0.78 & 1.01 & 0.42 & 1.07 & 0.07 & 0.04 & 0.07 \\
& $(0.25)$ & $(0.84)$ & $(0.55)$ & $(0.59)$ & $(0.08)$ & $(0.15)$ & $(0.07)$ \\
\hline $2-2005$ & 0.26 & 1.09 & 0.45 & 1.12 & 0.07 & 0.05 & 0.06 \\
& $(0.30)$ & $(1.35)$ & $(0.58)$ & $(0.68)$ & $(0.08)$ & $(0.14)$ & $(0.06)$ \\
\hline
\end{tabular}

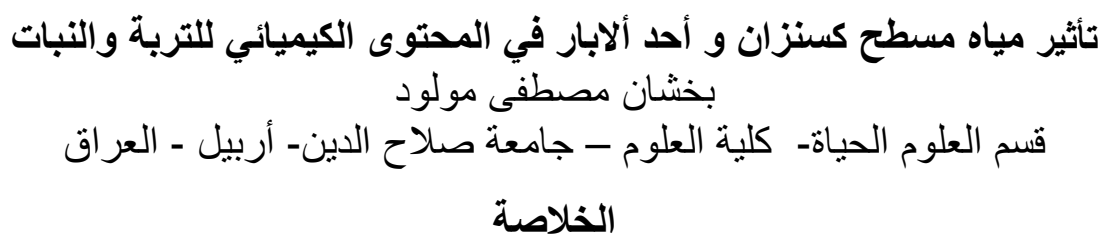


تم جمع العينات الثهرية من منطقة كسنزان لكل من التربة السطحية ونبات اليوكالبتوس و و

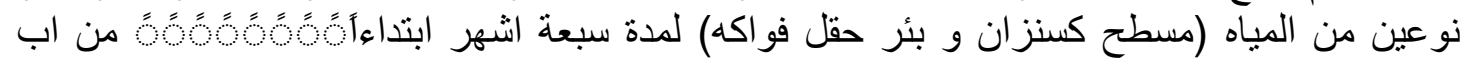

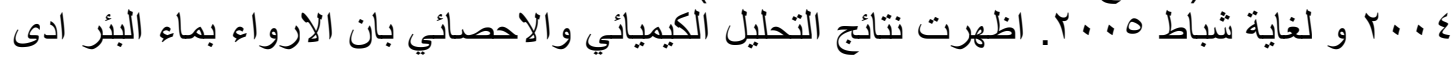

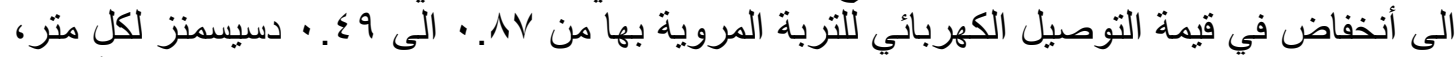

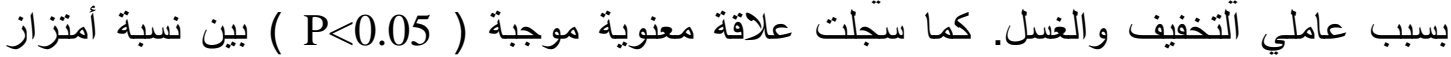

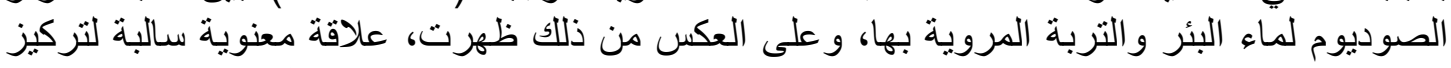

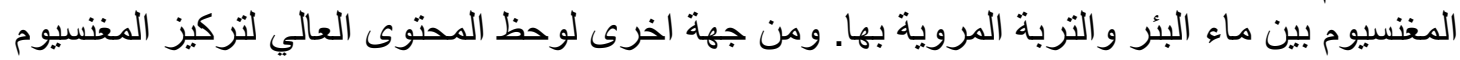
والمحتوى الواطيء لتركيز البوتاسيوم في نبات اليوكالبتوس المروي بماء البرئ البئر مقارنة بماء مسطح كسنز ان.

\section{REFERNCES}

Abu-Thahe, Y.M. (1989). Practical plant nutrition. Ministry of Higher Education and Scientific Research. Baghdad. Iraq.

Al-Barazingy,Y.O. (1995). Phycological study within Erbil province. M.Sc. thesis. University of Salahaddin- Hawler,Iraq.

Bapeer, U.H.K. (2004). Ecological study on the distribution of algae in different aquatic habitats within Erbil province. Ph.D. thesis. University of Salahaddin-Hawler, Iraq.

Dohuki,M.S.S. (1997). Classification of some wells and springs water in Dohuk governorate for irrigation and drinking purposes. M.Sc. thesis. University of Salahaddin-Hawler, Iraq.

Esmael, A.U. (1986). Limitation of some ground water in Erbil plain for different uses. M.Sc. thesis. University of Salkahaddin-Erbil.Iraq.

Esmael, A.U.; Maulood, P.M. and Shekha, Y.A. (2007). Evaluate Kasnazan impoundment water for irrigation purposes. J. Education and Sciense, the first conference on Biology, 20(2)47-56.

Goran,S.M.A. (2006). Limonological and non-diatom phytoplankton composition of Dilopa spring and Kasnazan impoundment,Hawler, Kurdistan Region of Iraq. M.Sc.thesis. University of SalahaddinHawler,Iraq.

Guo L,B. (2003). Sims,R.E. Soil response to eucalyptus tree planting and meet work effluent irrigation in a short rotation forest regime in New Zealand. Journal Bioresource Technology. 87 (3): 7-341.

Page,D.D.E. (1982). Ensmingger,L.E. and Clark,F.E. Methods of soil analysis. Part2. American Society Agriculture. Madison,Wisconson.USA.

Rasheed,R.O. (1994). A limnological study on some water system in Erbil province. M.Sc. thesis. University of Salahaddin-Hawler,Iraq.

Ryan,J. ; Estefon,G. and Rashid, A. (2001). Soil and plant analysis laboratory manual. $2^{\text {nd }}$ Edition. National Agriculture Research-Center. Islamabad, Pakistan.

Shirokova,Y. ; Forkutsa,I. and Sharafutdinova, N. (2000). Use of electrical conductivity instead of soluble salts for soil salinity monitoring in central Asia. Journal Irrigation and Drainage System. 14:199-205.

Taiz,L. and Zeiger,E. (2006). Plant physiology. $4^{\text {th }}$.ed. Sinauer Associates, Inc. USA.

Toma,J.J. (2004 ). Weekly and spatial variation of physico-chemical variables and algal compositions in Kasnazan impoundment, Erbil, Iraq. Journal of Babylon University, Iraq. 10(3). 
United State Salinity Laboratory Staff. (1954). Diagnosis and improvement of saline and alkali soils.

Van Hoorn, J.W. (1970). Irrigation and drainage . Paper 7. Salinity Seminar. Baghdad. FAO. Rome. 